Molecules 2002, 7, 412-419

molecules

ISSN 1420-3049

http://www.mdpi.org

\title{
Microwave Assisted Regioselective Bromomethoxylation of Alkenes Using Polymer Supported Bromine Resins
}

\author{
Geetha Gopalakrishnan, ${ }^{1, *}$ Viswanathan Kasinath, ${ }^{1}$ N.D.Pradeep Singh, ${ }^{1}$ V.P.Santhana \\ Krishnan, ${ }^{1}$ K.Anand Solomon, ${ }^{2}$ and S.S.Rajan ${ }^{2}$ \\ ${ }^{1}$ Centre for Natural Products, SPIC Science Foundation, 64 Mount Road, Guindy, Chennai 600032 , \\ India. \\ ${ }^{2}$ Department of Crystallography and Biophysics, University of Madras, Chennai, 600 025, India.
}

*Author to whom the correspondence should be addressed; e-mail: geethagopal@hotmail.com

Received: 6 March 2002; in revised form: 30 April 2002 / Accepted: 30 April 2002 / Published: 31 May 2002

\begin{abstract}
A facile regio- and chemoselective bromomethoxylation of alkenes under microwave irradiation conditions employing a new polymer supported brominechloride resin is reported. The resin is prepared from the commercially available chloride resin by a simple one step procedure.
\end{abstract}

Keywords: Bromomethoxylation; polymer supported bromine resins; microwave irradiation

\section{Introduction}

Application of polymer-supported reagents in organic synthesis has grown over the years due to its convenient handling and easy work up procedures [1]. The importance of these reagents is much felt in the new approach of combinatorial synthesis, where high throughput solution phase synthesis is designed with polymer supported reagents [2]. The present study demonstrates the stability of the new polymer supported bromine chloride resin and the previously reported perbromide resin [3] to microwave irradiation conditions. A facile regio and chemo selective bromomethoxylation of a variety of alkenes employing the above reagents is reported, including examples from natural sources. 


\section{Results and Discussion}

In our continuing study on the functionalisation of isoprenyl coumarins [4] directed towards identifying bioactive leads, the bromomethoxylation of osthol (9) was attempted. The available cohalogenating reagents, namely bromine in methanol [5], NBS/methanol [6] and N-bromoacetamide/ methanol [7] on reaction with 9, either resulted in multiple products or posed problems during isolation of the desired product. Hence, an attempt was made to apply the polymer supported perbromide resin (I), for the desired reaction. Resin I has been reported to effect the bromination of phenols [8] and ketones [9]. When 9 was treated with resin $\mathbf{I}$ in methanol, at either room temperature or under reflux conditions, bromomethoxylated product along with the corresponding bromohydroxy compound were obtained. The formation of bromohydroxy compound was rationalized as due to a demethylation of the bromomethoxy compound by the liberated $\mathrm{HBr}$ during the course of the reaction. However, when the reaction mixture was subjected to microwave irradiation (MWI), the reaction proceeded quantitatively within 30 seconds yielding the desired bromomethoxy compound. Hence a detailed study employing polymer supported reagents under MWI was taken up with other alkenes.

The preparation of the perbromide resin has been reported [8] from the commercially available IRA $400 ®$ chloride exchange resin by successive treatments with aq. $\mathrm{NaOH}$, aq. $\mathrm{HBr}$ and $\mathrm{Br}_{2} / \mathrm{CCl}_{4}$ as depicted in Scheme 1. An alternative and hitherto unreported polymer supported bromine resin, the brominechloride resin (II) was successfully prepared by a simple one step procedure from the commercially available chloride resin. (Scheme 1)

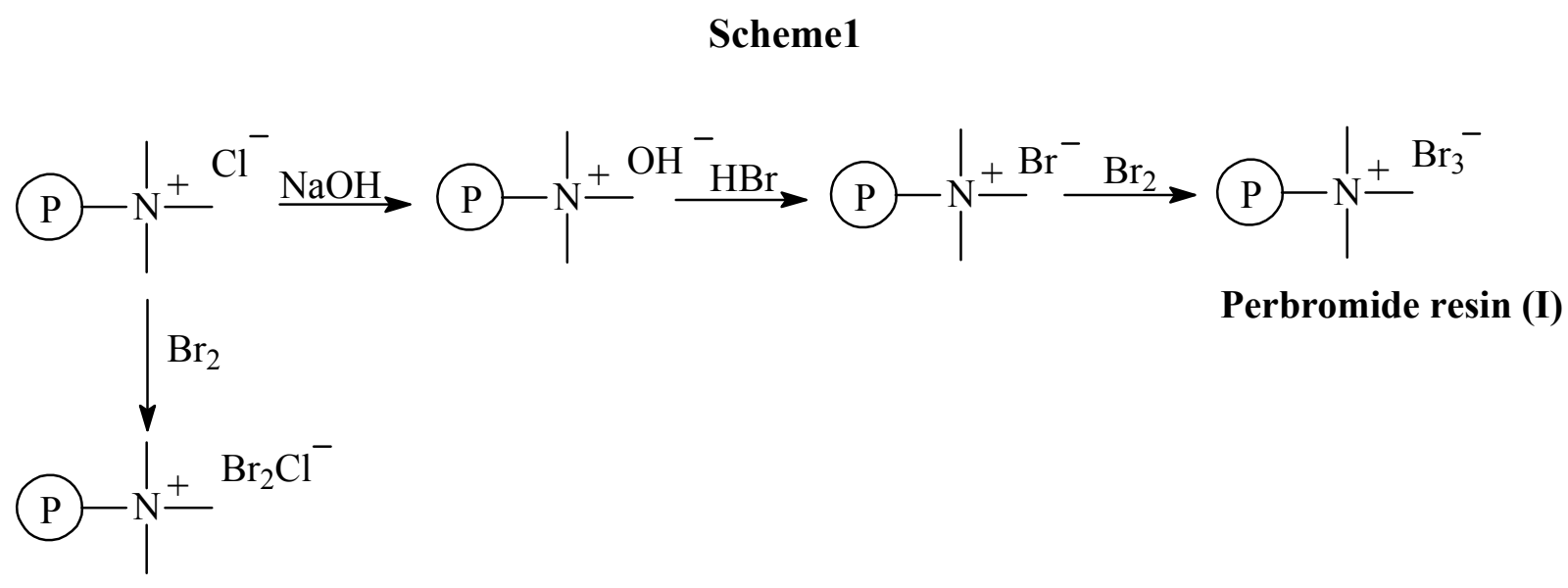

Brominechloride resin (II)

Both the resins I and II were found to possess the same amount of bromine and there was no appreciable change in the bromine content of these resins even after irradiating under MWI for 5 minutes as evident from the results reported in Table 1. 
Table1: Bromine content of the resins after MWI*.

\begin{tabular}{cccc}
\hline S.No & $\begin{array}{c}\text { MWI } \\
(\mathbf{m i n})\end{array}$ & $\begin{array}{c}\text { Perbromide resin } \\
(\mathbf{m m o l} / \mathbf{g})\end{array}$ & $\begin{array}{c}\text { Brominechloride resin } \\
(\mathbf{m m o l} / \mathbf{g})\end{array}$ \\
1 & 0 & 1.656 & 1.605 \\
2 & 0.5 & 1.652 & 1.592 \\
3 & 2 & 1.628 & 1.571 \\
4 & 5 & 1.601 & 1.518 \\
\hline \multirow{2}{*}{ * 2g of the resin in 10 ml of methanol was irradiated in a modified microwave } \\
\multicolumn{7}{r}{ oven [10] and then analyzed for bromine content. MWI- microwave irradiation. }
\end{tabular}

Various substituted alkenes: monosubstituted-1-decene (1); disubstituted- isoprenol (2); methyl angolensate, (3); carvone, (4); azadirachtin-A, (5); trisubstituted- prenol, (6); 2-(3-methylbutenyl)-1methoxybenzene, (7); 4-(3-methylbutenyl)-1-methoxy-benzene, (8); osthol, (9); tetra substituted - 2,3dimethylbutene, (10), yielded the respective bromomethoxyalkanes in moderate to good yields on treatment with the resins I or II in methanol under microwave irradiation in a shorter reaction time (Scheme 2).

Scheme 2
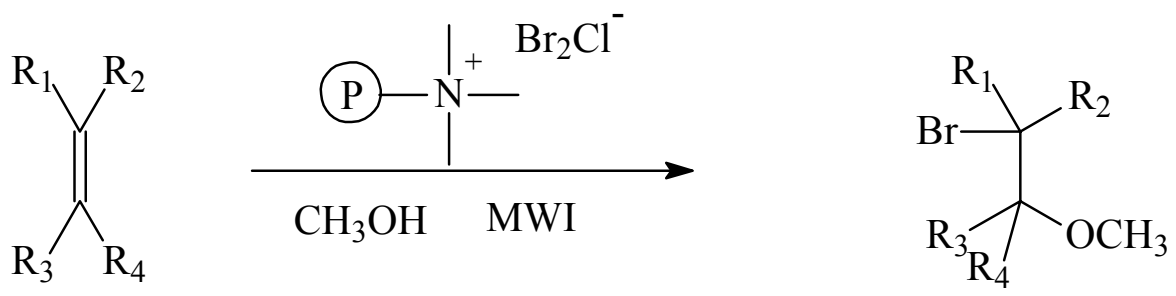

The results are presented in the following table. (Table 2)

Table 2: Bromomethoxylation of alkenes with perbromide resin (I) and brominechloride resin (II) under microwave irrdiation.

\begin{tabular}{|c|c|c|c|c|c|c|}
\hline \multirow[t]{2}{*}{ Alkene } & \multirow{2}{*}{\multicolumn{2}{|c|}{ Product }} & \multicolumn{2}{|c|}{$\begin{array}{l}\text { Perbromide } \\
\text { resin (I) }\end{array}$} & \multicolumn{2}{|c|}{$\begin{array}{l}\text { Brominechloride } \\
\text { resin (II) }\end{array}$} \\
\hline & & & Time(min) & Yield(\%) & Time(min) & Yield(\%) \\
\hline 1 & 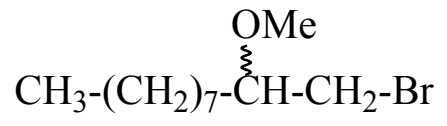 & $\mathbf{1 a}[11]$ & 5 & 68 & 5 & 57 \\
\hline 2 & $\begin{array}{c}\mathrm{OMe} \\
\mathrm{Br}-\mathrm{CH}_{2}-\stackrel{\xi}{\mathrm{C}}\left(\mathrm{CH}_{3}\right)-\mathrm{CH}_{2}-\mathrm{CH}\end{array}$ & $\mathbf{2 a}$ & 5 & 61 & 5 & 65 \\
\hline
\end{tabular}




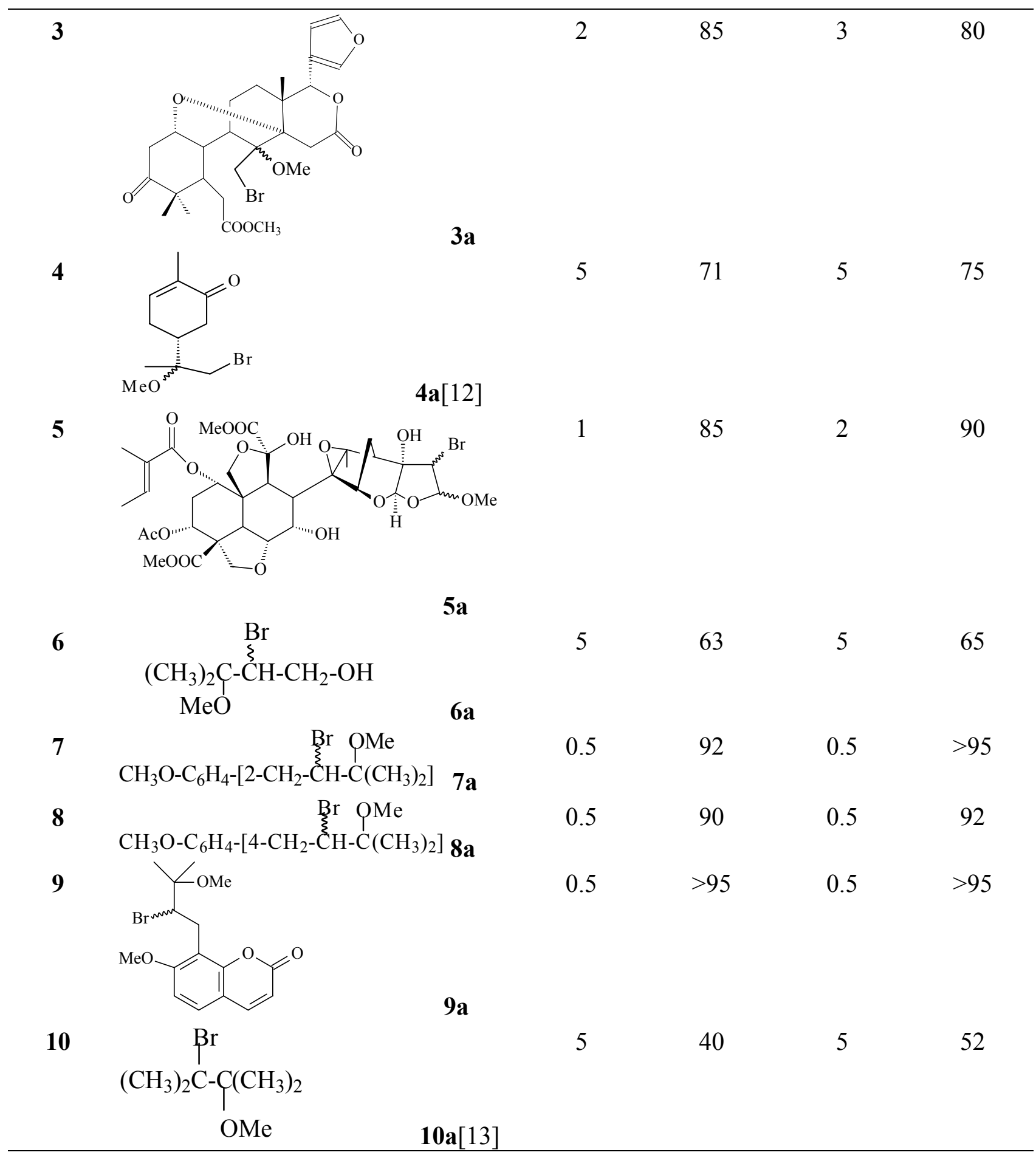

All the products were fully characterized by their spectral data $\left({ }^{1} \mathrm{H}-,{ }^{13} \mathrm{C}-\mathrm{NMR}\right.$ and MS). For one of the products (9a), the structure was also solved by X-ray crystallography [14] (Figure 1). 
Figure 1: ORTEP diagram of compound 9a drawn at 30\% probability.

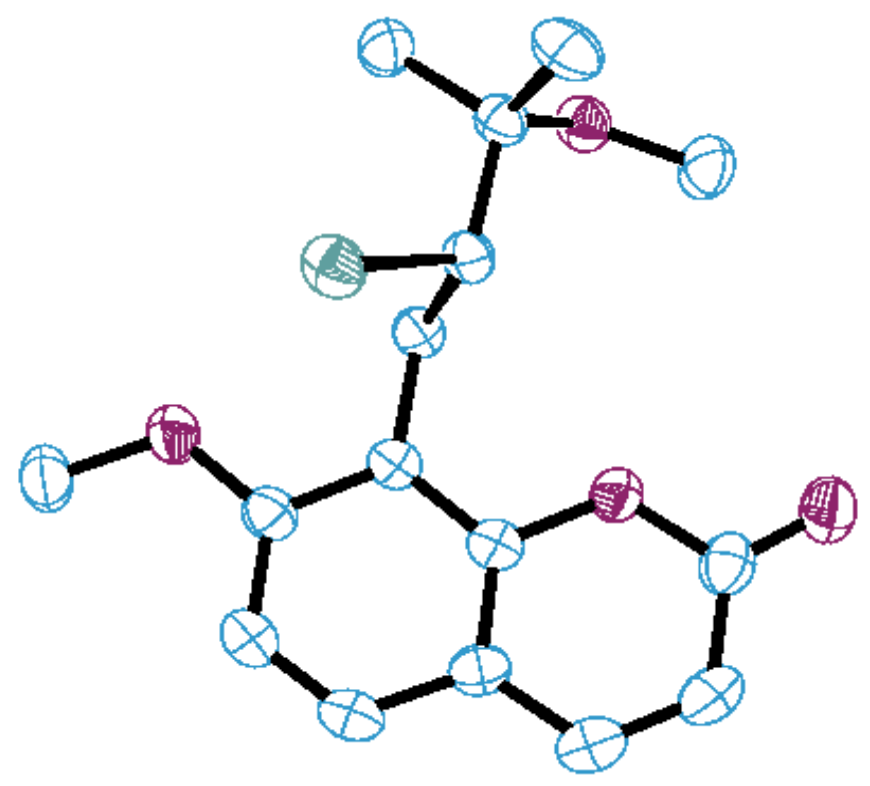

The reaction was found to be regioselective, following Markovnikov's addition rules, and also chemoselective as exemplified in the reaction of alkenes $\mathbf{4}$ and $\mathbf{5}$ where only the isolated double bonds were bromomethoxylated and the conjugated double bonds were inert to the reaction conditions. All the natural products considered for the present study $(\mathbf{3}, \mathbf{4}, \mathbf{5}, \mathbf{9}$ and 10) gave high yields of the corresponding bromomethoxy compound in a very short reaction time. Successive washings of the resin with methanol, acetonitrile and chloroform and then passing bromine in $\mathrm{CCl}_{4}$ through the resin, regenerated the polymer supported bromine reagent.

\section{Conclusions}

The present study illustrates a highly regio and chemoselective functionalisation of alkenes at short reaction times by the application of MWI to polymer supported reagents.

\section{Acknowledgements}

The authors thank CSIR, New Delhi for financial assistance.

\section{Experimental}

\section{General}

A modified domestic microwave oven (IFB-Megatron, wattage-1100W, power max-750W, voltage- $230 \mathrm{~Hz}$, frequency-2450MHz) equipped with a refluxing unit was used. NMR spectra were recorded on a Bruker $200 \mathrm{MHz}$ instrument using TMS as the internal reference for both ${ }^{1} \mathrm{H}$ - and 
${ }^{13} \mathrm{C}$-NMR experiments. $\mathrm{CDCl}_{3}$ was used as the solvent. Chemical shifts are given in terms of parts per million ( $\delta$ scale). MS analyses were recorded on a Shimadzu QP 5000 instrument. A DB-1 capillary column (30 meters length, 0.25 i.d) was used for all the GC analysis. Mass spectra were recorded in the EI mode. Precoated thin layer chromatography plates (E-merck, Germany, Keiselgel $60 \mathrm{~F}_{254}, 0.2 \mathrm{~mm}$ thickness, coated on aluminum sheets) were used. Column chromatography was performed using silica gel (60-120 mesh). IRA $400 \mathrm{Cl}^{-}{ }^{\circledR}$ (product equivalent to Amberlyst-26, Fluka) was obtained from SD Fine Chemicals Ltd., India.

Preparation of Brominechloride resin (II). Oven dried IRA-400® $\left(25 \mathrm{~g} ; \mathrm{Cl}^{-}\right.$form) was packed in a column plucked with cotton on both the ends. A solution of bromine $(8 \mathrm{~g} ; 0.05 \mathrm{~mol})$ in $\mathrm{CCl}_{4}(100 \mathrm{ml})$ was passed through the column till the color of the resin turned yellow-orange. Then the column was washed with $\mathrm{CCl}_{4}$ till all the free bromine was washed off and the washings were colorless. The resin beads were dried in a vacuum oven at $60^{\circ} \mathrm{C}$ for 3 hours.

Bromomethoxylation of alkenes: general procedure. To a solution of alkene (1 mmol) in methanol $(10 \mathrm{~mL})$, was added the polymer supported reagents I or II ( $1 \mathrm{~g}$, containing $0.256 \mathrm{~g}$ of bromine, 1.6 mmol) and irradiated in a modified domestic microwave oven fitted with a refluxing unit. After the reaction was complete (TLC), the reaction mixture was filtered; the resin was washed with methanol (3 $\mathrm{x} 10 \mathrm{ml}$ ). The filtrate was evaporated and the residue was purified by vacuum liquid chromatography (Si gel 230-400 mesh, eluent-Ethyl acetate: hexane) for the compounds 3a, 5a, 7a, 8a and 9a and by column chromatography (Si gel 60-120 mesh, eluent: ethyl acetate-hexane) for 1a, 2a, 4a, 6a and 10a to yield pure products respectively.

\section{Spectral data:}

1-Bromo-4-hydroxy-2-methoxy-2-methylbutane (2a): $\quad \mathrm{MF}-\mathrm{C}_{6} \mathrm{H}_{13} \mathrm{BrO}_{2}$ : LRMS(EI;m/z)-196, 198 (5.68\% : 5.67\%). ${ }^{1} \mathrm{H}-\mathrm{NMR}: 1.20(3 \mathrm{H} ; \mathrm{s}), 1.93(1 \mathrm{H} ; \mathrm{s}), 2.14(2 \mathrm{H} ; \mathrm{t}, \mathrm{J}=5.7 \mathrm{~Hz}), 3.36(3 \mathrm{H} ; \mathrm{s}), 3.42(2 \mathrm{H}$; s), 4.21(2H; t, J=5.7Hz). ${ }^{13} \mathrm{C}-\mathrm{NMR}: 24.4,29.6,43.7,57.4,59.6,61.2$.

3-0-bromo-8-methoxymethylangolensate (3a): MF- $\mathrm{C}_{27} \mathrm{H}_{35} \mathrm{BrO}_{8}: \mathrm{LRMS}(\mathrm{EI} ; \mathrm{m} / \mathrm{z})-566,568$ (2.68\% : 2.67\%); ${ }^{1} \mathrm{H}-\mathrm{NMR}: 0.86(3 \mathrm{H}, \mathrm{s}), 0.90(3 \mathrm{H}, \mathrm{s}), 1.08(3 \mathrm{H}, \mathrm{s}), 1.14(1 \mathrm{H}, \mathrm{m}), 1.17(3 \mathrm{H}, \mathrm{s}), 1.21(1 \mathrm{H}, \mathrm{m})$, $1.28(1 \mathrm{H}, \mathrm{m}), 2.16(1 \mathrm{H}, \mathrm{m}), 2.18(1 \mathrm{H}, \mathrm{m}), 2.25(1 \mathrm{H}, \mathrm{m}), 2.56(1 \mathrm{H}, \mathrm{m}), 2.85(1 \mathrm{H}, \mathrm{m}), 2.89(1 \mathrm{H}, \mathrm{m}), 2.94$ $(1 \mathrm{H}, \mathrm{m}), 3.18(3 \mathrm{H}, \mathrm{s}), 3.31(2 \mathrm{H}, \mathrm{s}), 3.46(1 \mathrm{H}, \mathrm{dd}, \mathrm{J}=6.1 \mathrm{~Hz}$ and $3.7 \mathrm{~Hz}), 3.70(3 \mathrm{H}, \mathrm{s}), 5.63(1 \mathrm{H}, \mathrm{s}), 6.29$ $(1 \mathrm{H}, \mathrm{d}, \mathrm{J}=6.1 \mathrm{~Hz}), 7.26(1 \mathrm{H}, \mathrm{s}), 7.39(1 \mathrm{H}, \mathrm{dd}, \mathrm{J}=6.2$ and $3.4 \mathrm{~Hz}) ;{ }^{13} \mathrm{C}-\mathrm{NMR}: 23.3,28.8,32.8,33.6$, $35.2,39.5,42.8,43.9,47.8,49.3,51.9,57.2,73.1,78.1,79.5,139.5,142.5,146.6,169.5,173.5,212.7$, 26.5, 21.4, 21.2, 13.5. 


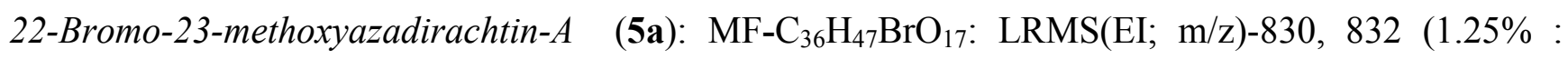
1.27\%). ${ }^{1} \mathrm{H}-\mathrm{NMR}: 1.33(1 \mathrm{H}, \mathrm{d}, \mathrm{J}=5.8 \mathrm{~Hz}), 1.73(1 \mathrm{H}, \mathrm{d}, 5.7 \mathrm{~Hz}), 1.74(3 \mathrm{H}, \mathrm{s}), 1.78$ (3H, dq), $1.85(3 \mathrm{H}$, dq), $1.95(3 \mathrm{H}, \mathrm{s}), 2.01(3 \mathrm{H}, \mathrm{s}), 2.15(1 \mathrm{H}, \mathrm{m}), 2.31(1 \mathrm{H}, \mathrm{m}), 2.38(1 \mathrm{H}, \mathrm{d}, \mathrm{J}=7.1 \mathrm{~Hz}), 2.56(1 \mathrm{H}, \mathrm{m}), 2.58$ $(1 \mathrm{H}, \mathrm{m}), 2.84(1 \mathrm{H}, \mathrm{d}, \mathrm{J}=7.1 \mathrm{~Hz}), 2.90(1 \mathrm{H}, \mathrm{bs}), 3.33(1 \mathrm{H}, \mathrm{s}), 3.38(3 \mathrm{H}, \mathrm{s}), 3.37(1 \mathrm{H}, \mathrm{d}, \mathrm{J}=6.5 \mathrm{~Hz}), 3.64$ $(1 \mathrm{H}, \mathrm{d}, \mathrm{J}=6.1 \mathrm{~Hz}), 3.68(3 \mathrm{H}, \mathrm{s}), 3.75(1 \mathrm{H}, \mathrm{d}, \mathrm{J}=6.5 \mathrm{~Hz}), 3.79(3 \mathrm{H}, \mathrm{s}), 4.09(1 \mathrm{H}, \mathrm{d}, \mathrm{J}=6.1 \mathrm{~Hz}), 4.17(1 \mathrm{H}, \mathrm{d}$, $\mathrm{J}=3.8 \mathrm{~Hz}), 4.58(1 \mathrm{H}, \mathrm{dd}, \mathrm{J}=4.5$ and $7.8 \mathrm{~Hz}), 4.67(3 \mathrm{H}, \mathrm{s}), 4.76(1 \mathrm{H}, \mathrm{d}, \mathrm{J}=3.7 \mathrm{~Hz}), 4.77(1 \mathrm{H}, \mathrm{d}, \mathrm{J}=4.5 \mathrm{~Hz})$, $5.50(1 \mathrm{H}, \mathrm{dd}, 3.8$ and $7.7 \mathrm{~Hz}), 6.94(1 \mathrm{H}, \mathrm{dd}, \mathrm{J}=6.1$ and $7.4 \mathrm{~Hz}):{ }^{13} \mathrm{C}-\mathrm{NMR}: 11.9,14.3,18.1,20.8,21.8$, 29.6, 37.0, 45.6, 47.6, 50.2, 52.3, 52.7, 53.3, 57.4, 57.6, 66.9, 67.7, 68.6, 69.1, 70.3, 73.0, 74.2, 76.3, $78.2,102.7,104.1,110.3,128.5,137.7,166.2,169.6,171.7,173.3$.

2-Bromo-3-methoxy-3methylbutanol (6a): MF- $\mathrm{C}_{6} \mathrm{H}_{13} \mathrm{BrO}_{2}$ : LRMS(EI; m/z)-196, 198 (6.32\% : 6.32\%). ${ }^{1} \mathrm{H}-\mathrm{NMR}: 1.7$ (3H; s), $1.82(3 \mathrm{H} ; \mathrm{s}), 2.24(1 \mathrm{H} ; \mathrm{bs}), 3.40(3 \mathrm{H}, \mathrm{s}), 3.91(1 \mathrm{H} ; \mathrm{t}, \mathrm{J}=7.3 \mathrm{~Hz}), 4.49(2 \mathrm{H} ; \mathrm{d}$, $\mathrm{J}=7.2 \mathrm{~Hz}) ;{ }^{13} \mathrm{C}-\mathrm{NMR}: 29.4,35.2,65.8,68.8,83.7$.

2-(2-Bromo-3-methoxy-3-methylbutyl)-1-methoxybenzene (7a): $\mathrm{MF}-\mathrm{C}_{13} \mathrm{H}_{19} \mathrm{BrO}_{2}$ : $\mathrm{LRMS}(\mathrm{EI} ; \mathrm{m} / \mathrm{z})-286$, 288 (4.65\% : 4.64\%); ${ }^{1} \mathrm{H}-\mathrm{NMR}: 1.26(6 \mathrm{H} ; \mathrm{s}), 2.73(2 \mathrm{H} ; \mathrm{m}), 3.25(3 \mathrm{H} ; \mathrm{s}), 3.78(3 \mathrm{H} ; \mathrm{s}), 4.1(1 \mathrm{H} ; \mathrm{dd}, \mathrm{J}=$ 6.1 and $3.9 \mathrm{~Hz}), 6.8(2 \mathrm{H} ; \mathrm{m}), 7.2(2 \mathrm{H} ; \mathrm{m}) ;{ }^{13} \mathrm{C}-\mathrm{NMR}: 21.2,22.4,27.0,49.6,55.7,59.7,77.4,115.6$, $119.6,127.1,142.8$.

4-(2-Bromo-3-methoxy-3-methylbutyl)-1-methoxybenzene (8a): MF- $\mathrm{C}_{13} \mathrm{H}_{19} \mathrm{BrO}_{2}$ : LRMS (EI; m/z)286, 288 (7.51\% : 7.51\%); ${ }^{1} \mathrm{H}-\mathrm{NMR}: 1.21$ (6H; s), $2.86(2 \mathrm{H} ; \mathrm{m}), 3.30(3 \mathrm{H} ; \mathrm{s}), 3.83$ (3H; s), 4.26(1H; $\mathrm{dd}, \mathrm{J}=6.3$ and $3.9 \mathrm{~Hz}), 6.8(2 \mathrm{H} ; \mathrm{d}, \mathrm{J}=7.8 \mathrm{~Hz}), 7.3(2 \mathrm{H} ; \mathrm{d}, \mathrm{J}=7.8 \mathrm{~Hz}) ;{ }^{13} \mathrm{C}-\mathrm{NMR}: 21.4,22.6,27.3,48.7$, $55.9,59.9,77.2,115.8,120.1,127.2,143.4$.

7-Methoxy-8-(2-bromo-3-methoxy-3-methylbutyl)coumarin (9a): MF- $\mathrm{C}_{16} \mathrm{H}_{19} \mathrm{BrO}_{4}$ : LRMS (EI; m/z)354, 356 (3.35\% : 3.36\%); ${ }^{1} \mathrm{H}-\mathrm{NMR}: 1.32(3 \mathrm{H} ; \mathrm{s}), 1.44(3 \mathrm{H} ; \mathrm{s}), 3.40(3 \mathrm{H} ; \mathrm{s}), 3.52(2 \mathrm{H} ; \mathrm{m}), 3.96(3 \mathrm{H}$; s), $4.61(1 \mathrm{H} ; \mathrm{dd}, \mathrm{J}=4.5$ and 5.2Hz), $6.20(1 \mathrm{H} ; \mathrm{d}, \mathrm{J}=6.9 \mathrm{~Hz}), 6.85(1 \mathrm{H} ; \mathrm{d}, \mathrm{J}=7.4 \mathrm{~Hz}), 7.42(1 \mathrm{H} ; \mathrm{d}$, $\mathrm{J}=6.9 \mathrm{~Hz}), 7.6(1 \mathrm{H} ; \mathrm{d}, \mathrm{J}=7.4 \mathrm{~Hz}) ;{ }^{13} \mathrm{C}-\mathrm{NMR}: 22.3,23.6,26.8,49.6,56.0,60.5,77.2,107.4,113.0,115.6$, 119.5, 127.1, 143.7, 160.6; Crystal data: [14] Triclinic, P-1, a = 10.280 (2) $\AA, \mathrm{b}=10.497$ (4) $\AA, \mathrm{c}=$ 16.175 (4) $\AA, \alpha=75.91(2)^{\circ}, \beta=80.81(2)^{\circ}, \gamma=68.00(3)^{\circ}, V=1565.1$ (8) $\AA^{3}, \lambda=1.54184 \AA, R=$ 0.0638 . 


\section{References and Notes}

1. Shuttleworth, S. J.; Allin, S. M.; Wilson, R. D.; Nasturica, D. Synthesis, 2000, 1035-1074.

2. $\quad$ Bhalay, G.; Dunstan, A.; Glen, A. Synlett., 2000, 12, 1846-1859.

3. Akelah, A.; Sherrington, D.C. Chem. Rev., 1981, 81, 557-587.

4. Gopalakrishnan, G.; Kasinath, V.; Pradeep Singh, N.D.; Thirumurugan, R.; Sundararaj, S.S.; Shanmugam, G. Molecules, 2000, 5, 845-848.

5. Curtin, D. Y.; Meislich, E.K. J. Amer. Chem. Soc., 1952, 74, 5518-5520.

6. $\quad$ Dulcere, J. P.; Rodriguez, J.; Santelli, M.; Zahara, J. P. Tetrahedron Lett., 1987, 28, 2009-2012.

7. $\quad$ Buckles, R. E.; Filler, R.; Hilfman, L. J. Org. Chem., 1952, 233-242.

8. Smith, K.; James, M.; Matthews, I.; Bye, M. R. J. Chem. Soc. Perkin Trans. 1, 1992, $1877-$ 1878.

9. Cacchi, S.; Caglioti, L. Synthesis, 1979, 64-66.

10. Geetha Gopalakrishnan; Pradeep Singh, N.D.; Kasinath, V.; Sivarama Krishnan, M.; Malathi, R.; Rajan,S.S. Tetrahedron Lett, 2001, 42, 6577-6579.

11. 1-Bromo-2-methoxydecane (1a): CA: 64:11076a, 1965.

12. 5-(2-Bromo-1-hydroxy-1-methylethyl)-2-methylcyclohex-2-ene-1-one (4a): Calo,V.; Lopez,L.; Fiandanese,V.; Naso,F.; Ronzini,L. Tetrahedron Lett. 1978, 49, 4963-4966.

13. 2-Bromo-3-methoxy-2,3-dimethylbutane (10a): Olah, G.A.; Bollinger, J.M. J. Amer. Chem. Soc. 1967, 89, 4744-4746.

14. $\quad$ CCDC No: 173576.

Sample availability: Samples of compounds 1a, 2a, 4a, 6a, 7a, 8a, 9a and 10a are available from the authors

(C) 2002 by MDPI (http://www.mdpi.org). Reproduction is permitted for non commercial purposes. 